\title{
Emphysematous Pyelonephritis: Almost the Same Ancient Disaster
}

\author{
Metin Tas $^{1}$ and Ayse Veyhurda Dikmen ${ }^{2 *}$ \\ ${ }^{1}$ Urologist, MD, Ankara Özel Lokman Hekim Hastaneleri, Ankara, Turkey \\ ${ }^{2}$ Consultant urologist, M.D. Ankara, Turkey
}

*Corresponding author: Ayse Veyhurda Dikmen, Consultant urologist, M.D. Ankara, Turkey

\begin{abstract}
A 71 year-old male patient, who was diabetic and hypotensive, was admitted to emergency room with the complaints of high fewer, shortness of breath and general malaise. He was diagnosed to have acute renal failure, signs of uro-sepsis and emphysematous pyelonephritis with pathognomonic computerized tomography (CT) scans. After 7 days of follow-up with meropenem and teicoplanin treatment and hemodialysis, the patient was externed. This case was an emergent case of emphysematous pyelonephritis, which is a rare entity with a high incidence of mortality in modern clinical practice.
\end{abstract}

\section{Introduction}

Emphysematous pyelonephritis (EPN) is a kind of highly mortal renal infection that results in gas production in the renal parenchyma, collecting system, or perinephric tissue. It has been one of the most devastating clinical problems of urology with almost unpreventable results with a high mortality rate. As we all know, there are mostly well known predisposing risk factor such as diabetes, increased patient age, prolonged duration of untreated complicating urinary tract infections and accompanying health problems. As a matter of fact, poorest economic conditions and bad sanitation also provide the background for highly attenuating the success of treatment. Although we are facing new cases of EPN in a decreasing proportion, it is still one of the most terrifying clinical conditions, in which the rapid diagnosis with CT scans is a prominent mile-stone for the sake of the patient. A 71 year-old male patient, who was diabetic and hypotensive, was admitted to emergency room with the complaints of high fewer, shortness of breath and general malaise. He was thorougly examined and was diagnosed to have metabolic acidosis, tacycardia, tacypnea and signs of disseminated infection throughout the body. After clinical work-up, he was diagnosed to have left EPN and signs of uro-sepsis, since his computerized tomography (CT) scans were completely pathognomonic for the diagnosis. Because of increased blood levels of creatinine, he was also suffering from acute renal failure and urosepsis. He was accepted to intensive care unit with signs of septic shock. After 7 days of follow-up with meropenem and teicoplanin treatment and a limited number of hemodialysis. The patient recovered dramatically upon undergoing left renal percutaneous drainage was discharged 12 days after admission with a creatinine blood level of $1.5 \mathrm{mg} / \mathrm{dl}$. This case was an emergent case of EPN, which is a rare entity with a high incidence of mortality in modern clinical practice.

13 other cases of EPN and ephysematous cystitis that were reported between 1962 and 2017, were reviewed and discussed by Li S et.al in 2018 [1]. The overall mortality was 15.4\% (two of 13 patients), compared with $25 \%$ for emphysematous pyelonephritis alone reported in the literature [1]. Escherichia coli was isolated as the responsible pathogen [1]. As a matter of fact, uncontrolled diabetes is a severely complicating comorbidity, which can change the clinical progress completely. In this case, the patient was lucky enough to have an earlier CT scan, which can provide a higher rate of prevention of the patient from undergoing nephrectomy. Intensive care unit is also a prominent treatment opportunity for 
the patient with a facility of percutaneous renal drainage on time. Ziegelmuller et.al. [2] declared that radical nephrectomy being performed immediately was the optimal management in patients with acute emphysematous nephritis and urosepsis [2].

\section{References}

1. Shengxian Li, Jixue Wang, Jinghai Hu, Liang He, Chunxi Wang (2018) Emphysematous pyelonephritis and cystitis: A case report and literature review. J Int Med Res 46(7): 2954-2960.
2. Brigitte Katharina Ziegelmüller, Bernadett Szabados, Annabel Spek, Jozefina Casuscelli, Christian Stief, et al. (2018) Emphysematous pyelonephritis: Case report and literature overview. Urologia 85(3): 123-126.
(C) (P) This work is licensed under Creative

To Submit Your Article Click Here: Submit Article

DOI: $10.32474 /$ GJAPM.2020.03.000171

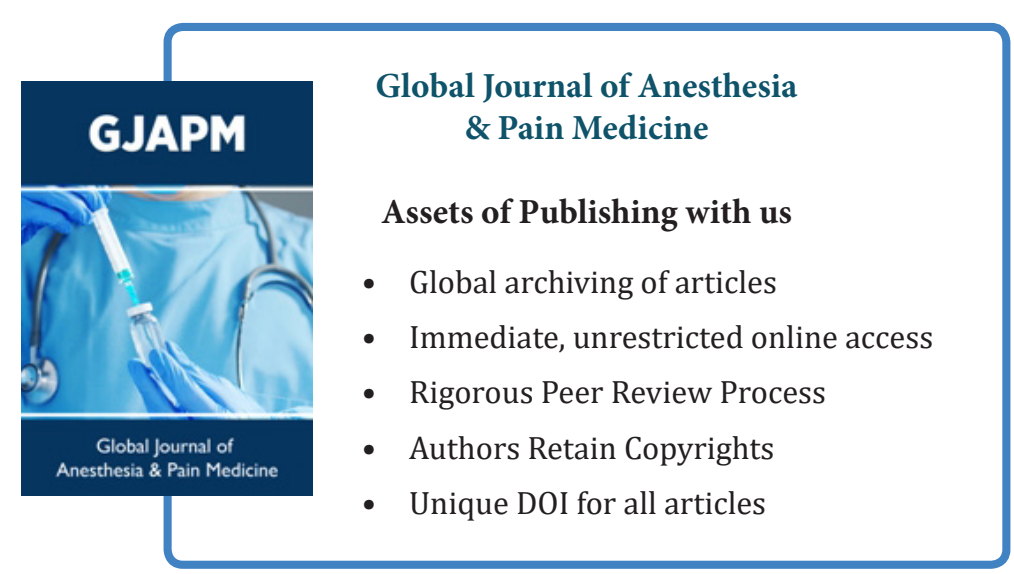

